\title{
Application Research of Virtual Desktop Infrastructure in the NCRE
}

\author{
Wang Naiguo,Zhu Xiangwei*,Zhang Hong,Wang \\ Jianzhong,Xie Ying,Rong Siwei \\ Suzhou Industrial Park Branch \\ Jiangsu Union Technical Institute \\ Suzhou, China \\ zxwook@163.com*corresponding author
}

\begin{abstract}
According to the specific requirements of environment of National Computer Rank Examination (NCRE), through the introduction horizon architecture of desktop virtualization software (WMware View) and introduction its method of installation, debugging, finally realizes the purpose of cloud computing examination of NCRE using desktop virtualization. Desktop virtualization in the application of the NCRE, can greatly reduce the work burden of computer room management personnel, improve the NCRE computer room deployment efficiency, and greatly satisfy the convenience of mobile learning requires, so students learning cannot be influenced by the computer room the constraints of physical location, greatly improve the passing rate of the NCRE.
\end{abstract}

Keywords-NCRE; cloud computing; desktop virtualization; mobile learing; computer room administrator

\section{INTRODUCTION}

The National Computer Rank Examination (NCRE) has been acknowledged widely since it was implemented over a decade ago, keeping rapid and healthy development. The NCRE is one of the important examinations held by the Ministry of education examination center. Many colleges and universities require students to have the NCRE Grade two or above certificate to at the time of graduation degree certificate or diploma, so the NCRE has become one of the important exams in Colleges and universities. Because of this, how to ensure the normal operation of two times a year to the NCRE of the hardware environment and software environment has become an important work of computer room administrator.

General hardware environment is better resolved, as long as computers, switches and network to work properly. More troublesome is the need to install a large number of test machines and make complicated settings, it is the primary problem for the computer room administrator how to install software of NCRE quickly and effectively into a lot of test machine.

Cloud computing is a model for enabling ubiquitous, convenient, on-demand network access to a shared pool of configurable computing resources (e.g., networks, servers, storage, applications, and services) that can be rapidly provisioned and released with minimal management effort or service provider interaction.

Cloud computing can be considered include the following levels of service: Infrastructure as a Service (IaaS), Platform as a Service (PaaS) and Software as a Service(SaaS)

\author{
Zuo Jinxiao \\ Jiangsu Foxti Information Technology \\ Suzhou, China \\ zuojx@foxti.cn
}

With the development of cloud computing, it is necessary to use the new technology in the NCRE. Installation of traditional NCRE spends a lot of manpower and time. Deployment and debugging the NCRE relatively tedious and each computer room is required to install a server. In order to prevent the failure of system and software needed to backup server, Server utilization is low. NCRE system based on cloud computing platform can change many disadvantages of the traditional examination system, conservation of resources, improve the safety, stability and using rate of the system hardware.

\section{HORIZON ARCHITECTURE}

Horizon (with View) allows organizations to deliver virtualized or remote desktops and applications through a single platform and support end users with access to all of their desktops and applications through a single unified workspace.

Fig .1 shows the relationship between the major components of View deployment.

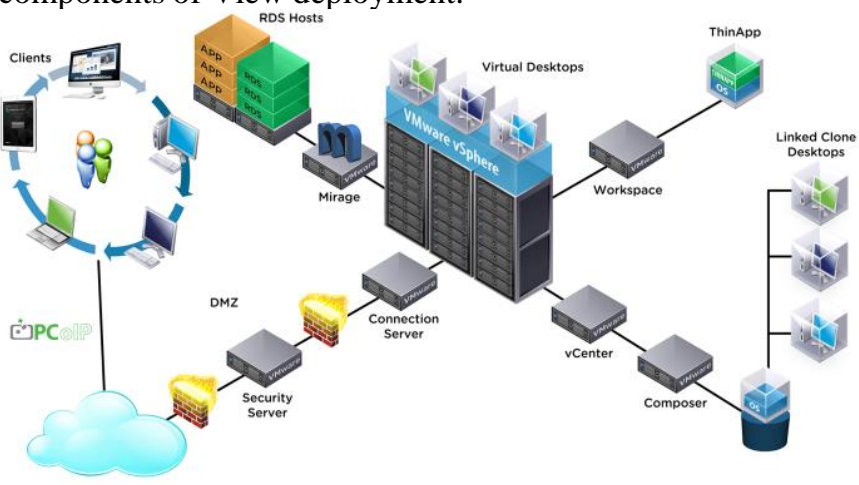

Figure 1. horizon architecture

End users start Horizon Client to $\log$ in to View Connection Server. This server, which integrates with Windows Active Directory, provides access to remote desktops hosted on a VMware vSphere server, a physical PC, or a Microsoft RDS host. Horizon Client also provides access to remote applications on a Microsoft RDS host.

\section{A. $\quad$ Client Devices}

A major advantage of using View is that remote desktops and applications follow the end user regardless of device or location. Users can access their personalized virtual desktop or remote application from a 
company laptop, their home PC, a thin client device, a Mac, or a tablet or phone.

End users open Horizon Client to display their remote desktops and applications. Thin client devices use View thin client software and can be configured so that the only application that users can launch directly on the device is View Thin Client. Repurposing a legacy PC into a thin client desktop can extend the life of the hardware by three to five years. For example, by using View on a thin desktop, you can use a newer operating system such as Windows 7 on older desktop hardware.

If you use the HTML Access feature, end users can open a remote desktop inside a browser, without having to install any client application on the client system or device.

\section{B. View Connection Server}

This software service acts as a broker for client connections. View Connection Server authenticates users through Windows Active Directory and directs the request to the appropriate virtual machine, physical PC, or Microsoft RDS host.

View Connection Server provides the following management capabilities:

- Authenticating users

- Entitling users to specific desktops and pools

- Assigning applications packaged with VMware ThinApp to specific desktops and pools

- Managing remote desktop and application sessions

- Establishing secure connections between users and remote desktops and applications

- Enabling single sign-on

- $\quad$ Setting and applying policies

Inside the corporate firewall, you install and configure a group of two or more View Connection Server instances. Their configuration data is stored in an embedded LDAP directory and is replicated among members of the group.

Outside the corporate firewall, in the DMZ, you can install and configure View Connection Server as a security server. Security servers in the DMZ communicate with View Connection Servers inside the corporate firewall. Security servers ensure that the only remote desktop and application traffic that can enter the corporate data center is traffic on behalf of a strongly authenticated user. Users can access only the resources that they are authorized to access.

Security servers offer a subset of functionality and are not required to be in an Active Directory domain. You install View Connection Server in a Windows Server 2008, Windows Server 2012, or Windows Server 2012 R2 server, preferably on a VMware virtual machine.

\section{VMware Horizon User Web Portal}

From a Web browser on a client device, end users can connect to remote desktops and applications through the browser, automatically start Horizon Client if it is installed, or download the Horizon Client installer.

\section{View Agent}

You install the View Agent service on all virtual machines, physical systems, and Microsoft RDS hosts that you use as sources for remote desktops and applications.
On virtual machines, this agent communicates with Horizon Client to provide features such as connection monitoring, virtual printing, View Persona Management, and access to locally connected USB devices.

If the desktop source is a virtual machine, you first install the View Agent service on that virtual machine and then use the virtual machine as a template or as a parent of linked clones. When you create a pool from this virtual machine, the agent is automatically installed on every remote desktop.

You can install the agent with an option for single signon. With single sign-on, users are prompted to $\log$ in only when they connect to View Connection Server and are not prompted a second time to connect to a remote desktop or application.

\section{E. View Administrator}

This Web-based application allows administrators to configure View Connection Server, deploy and manage remote desktops and applications, control user authentication, and troubleshoot end user issues.

When you install a View Connection Server instance, the View Administrator application is also installed. This application allows administrators to manage View Connection Server instances from anywhere without having to install an application on their local computer.

\section{F. View Composer}

You can install this software service on a VCenter Server instance that manages virtual machines or on a separate server. View Composer can then create a pool of linked clones from a specified parent virtual machine. This strategy reduces storage costs by up to 90 percent.

Each linked clone acts like an independent desktop, with a unique host name and IP address, yet the linked clone requires significantly less storage because it shares a base image with the parent.

Because linked-clone desktop pools share a base image, you can quickly deploy updates and patches by updating only the parent virtual machine. End users' settings, data, and applications are not affected.

Although you can install View Composer on its own server host, a View Composer service can operate with only one VCenter Server instance. Similarly, a VCenter Server instance can be associated with only one View Composer service.

\section{G. VCenter Server}

This service acts as a central administrator for VMware ESXi Servers that are connected on a network VCenter Server, provides the central point for configuring, provisioning, and managing virtual machines in the datacenter.

In addition to using these virtual machines as sources for virtual machine desktop pools, you can use virtual machines to host the server components of View including View Connection Server instances, Active Directory servers, Microsoft RDS hosts, and VCenter Server instances.

You can install View Composer on the same server as VCenter Server or on a different server. VCenter Server then manages the assignment of the virtual machines to physical servers and storage and manages the 
assignment of CPU and memory resources to virtual machines.

You can install VCenter Server either as a VMware virtual appliance or install VCenter Server in a Windows Server 2008 R2 server, a Windows Server 2012 server, or a Windows Server 2012 R2 server, preferably on a VMware virtual machine.

\section{DEMANDS OF THE WORK ENVIRONMENT NCRE}

Server-side settings: server-side installation examination management system, by clicking on the "generate directory", "generated users " and " directory authorization " button to create the new directory, create a new user (such as K01, k02, k03, etc.) and set the safety of the directory.

Exam-side settings: Each test machines need to create or modify a specific user name with administrator privileges, such as $\mathrm{k} 01, \mathrm{k} 02, \mathrm{k} 03$, established the user name and the server directory name (such as $\mathrm{K} 01, \mathrm{k} 02$, $\mathrm{k} 03$, etc.) correspond. Through the "map network drive" "command mapping a" K: "disk to the server's Ncre40 directory, after mapped the" $\mathrm{K}$ : " disk, the client test software system installed with NCRE, finally the user login server test whether the examination can the normal or not.

\section{TRADITIONAL SOLUTION}

Generally computer rooms have adopted management method of the hard disk protection card, first installed a test client machine, and then through the method of network spread installed the throughout computer room. In order to the safety of NCRE, NCRE requires each machine must use the specified username to login on the server, access to the server directory corresponding make examination. But the contents of machine are the same, because all the machines installed by the method of network spread. So you need to manually change the IP address, machine name, and add or modify the specified user name. Although the hard disk protection card has the function of modifies the machine name and IP address, at the same time by all users such as K01 to K99 are added to also can achieve a certain effect of user groups, but want to let each machine respectively with the specified username as the current default login name, also need the computer room administrator one by one set each machine, so computer room administrator must spend a lot of time in the setting of test machine, and a lot of computer room also needs to be restored to the daily class environment after the examination, which gives a lot of the tedious work for the computer room administrator, so the computer room administrator most concern how to simplify and deployment test machine quickly.

\section{APPLICATION OF DESKTOP VIRTUALIZATION TECHNOLOGY IN NCRE}

VMware View desktop virtualization technology provides great convenience for computer room management, the paper illustrate the application of the VMware View desktop virtualization technology for computer room of author unit in the NCRE.

\section{A. VMware View deployment planning and harwaer prepareing}

According to the VMware View deployment requirements and the NCRE examination environment requirements, planning the whole test environment as shown in the following table:

TABLE I. VMWARE VIEW DEPLOYMENT PLANNING

\begin{tabular}{|c|c|c|c|}
\hline Software Components & OS & Domain Name & IP Address \\
\hline ESXi Server & ESXi 5.5 & $\begin{array}{l}\text { Esxi1.sipits.com } \\
\text { Esxi2.sipits.com }\end{array}$ & $\begin{array}{l}192.168 .0 .101 \\
192.168 .0 .102\end{array}$ \\
\hline Connection Server & Server R2 x64 & $\begin{array}{l}\text { ViewconnSEV.sip } \\
\text { its.com }\end{array}$ & 192.168 .0 .105 \\
\hline $\begin{array}{l}\text { Domain Controller } \\
\text { DNS Server } \\
\text { DHCP Server } \\
\text { AD }\end{array}$ & Server R2 x64 & ad.sipits.com & 192.168.0.104 \\
\hline $\begin{array}{l}\text { VCenter } \\
\text { SQL Server } \\
\text { Composer Server }\end{array}$ & Server R2 x64 & vcenter.sipits.com & 192.168 .0 .103 \\
\hline $\begin{array}{l}\text { NCRE Client Virtual } \\
\text { Desktop }\end{array}$ & Win7 & sipits.com & $\begin{array}{l}192.168 .0 .200 \sim \\
192.168 .0 .253\end{array}$ \\
\hline
\end{tabular}

According to the plan of the TABLE I, need to prepare the server and storage hardware shown in Fig. 2.

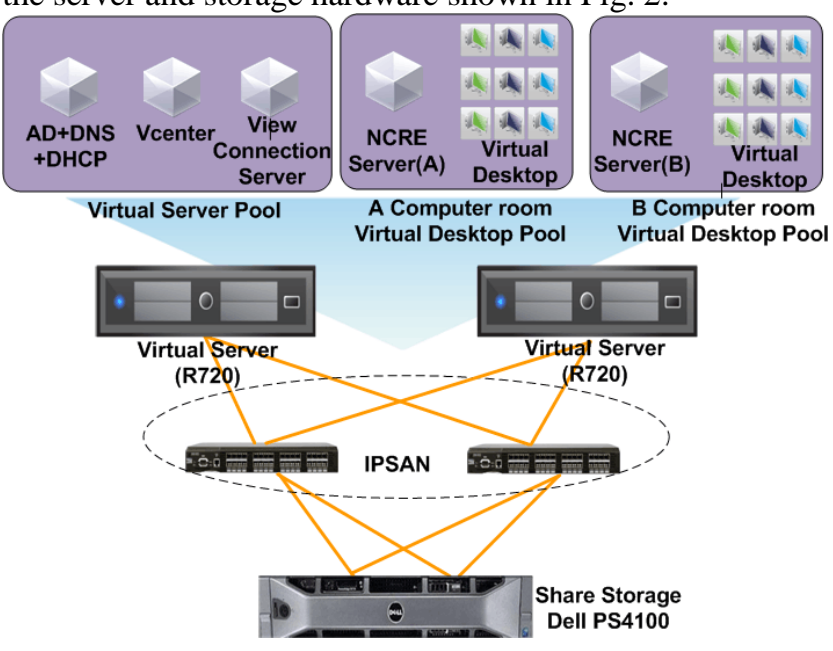

Figure 2. hardware prepare

\section{B. The installation of NCRE environment in Server}

\section{1) Install and configure VMware View Component} Software

First of all, in the two physical host are respectively installed on server virtualization software ESXi 5.5, and then virtualizes three virtual servers in the two physical host according to TABLE I planning requirements. It is ready for the installation of View Connection Server, Domain Controller, DNS Server, DHCP Server, AD, VCenter, SQL Server, Composer Server software components. Before install these software components, must be installed on three virtual servers with server 2008 R2 x64 operating system. After the software components are installed, according to the requirements of NCRE, you should configure DNS Server, DHCP Server, and AD etc.

2) Install the server software of NRCE

Virtualize a virtual server on a physical host. Install the server software of NRCE on this virtual server. After t the installation is completed, using complete cloning methods 
to generate a test server of NRCE. So two computer rooms test server of NRCE were ready.

\section{3) Install the client software of NRCE}

Virtualize a WIN7 32-bit desktop PC on a physical host. Install the client software of NCRE on this desktop PC. After the installation is completed, using linked-clone methods to generate thousands client machines of NCRE for students test service (due to the limitation of resources by two physical hosts, each computer room linked-clone only three client machines of NCRE). According NCRE requirements (user name K01 to K99), when linked-clone, set the machine name to fill in for $\mathrm{K}$, fill the starting number 01 , fill the end of the number 06 .

\section{EXPERIMENTAL RESULT}

As shown in Fig .3 is composed of two physical servers in the data center cluster, which uses VMware View desktop virtualization software components, to simulate the two computer rooms of the NRCE in the interface view of cluster and the host.

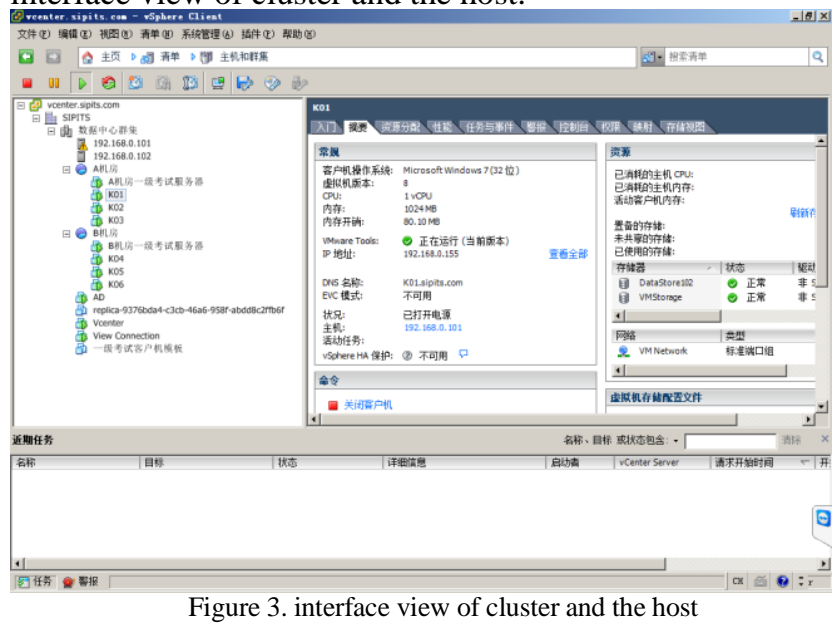

As shown in Fig .4 is the virtual machine resource mapping network graph, which shows all of the virtual machine and virtual machine storage and network connection diagram.

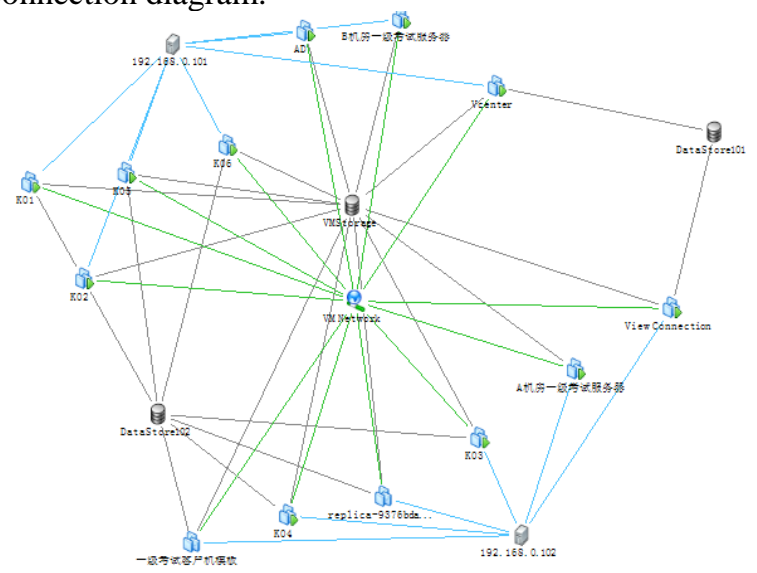

Figure 4. virtual machine resource mapping network graph

After finishing deployment of virtual Server and virtual desktop according Fig .2, install VMware Horizon Client software in Computer Room. The client software for accessing remote desktops and applications can run on a tablet, a phone, a Windows, Linux, or Mac PC or laptop, a thin client, and more.

Students can click the shortcut key of Horizon Client software, and then logging in, they can operate virtual desktop (remote desktops) as they sit at Server side.

\section{CONCLUSION}

Cloud desktop is successfully applied in the NCRE, overcome the traditional method requires one by one to modify login username and mapped drive and other work, you only need to configure once, does not need to be repeated to modify the computer name and user name, IP address and other work, thus greatly reducing the majority of the administrator's workload, to avoid duplication of effort, improve work efficiency.

More important is the NCRE using desktop virtualization, students can achieve the ubiquitous learning, and greatly satisfy the convenience of mobile learning requires. So students learning cannot be influenced by the computer room the constraints of physical location, greatly improve the pass rate of NCRE.

\section{REFERENCES}

[1] Han Guo-Yong; Zhao Hong-Luan; Liu Hao; Zhang Zhi-Jun; Xu Gong-Wen, Analysis and Design in NCRE Registration System Based on J2EE, 2013 International Conference on Communication Systems and Network Technologies (CSNT 2013). Proceedings, p 822-6, 2013

[2] Wang Chunhai,VMware Virtualization and Cloud Computing Applications Detailed[M].China Railway Press,2013

[3] Gu Jiongjiong, Cloud Computing Architecture Technologies \& Practice[M].Beijing: Tsinghua University Press,2014

[4] Agrawal, S.; Biswas, R.; Nath, A. Virtual Desktop Infrastructure in Higher Education Institution: Energy Efficiency as an Application of Green Computing, 2014 Fourth International Conference on Communication Systems and Network Technologies (CSNT), p 601-5, 2014

[5] Shalabh Agarwal, Shreya Goswami, Asoke Nath, Green Computing and Green Technology in e-Learning, Corporate, Business and IT Sectors, International Journal of Compute Applications(IJCA), Vol 76, No.7, (August), Pp. 35-41(2013).

[6] Muñoz, A.; Gonzalez, J.; Maña, A. A performance-oriented monitoring system for security properties in cloud computing applications[J], Computer Journal, v 55, n 8, p 979-94, Aug. 2012

[7] Sinha, Neelu; Khreisat, Laila .Cloud computing security, data, and performance issues. 2014 23rd Wireless and Optical Communication Conference (WOCC), p 6 pp., 2014

[8] Cindhamani, J.; Punya, N.; Ealaruvi, R.; Dhinesh Babu, L.D. ,An enhanced data security and trust management enabled framework for cloud computing systems,2014 Fifth International Conference on Computing, Communications and Networking Technologies (ICCCNT), p 5 pp., 2014

[9] Huang Jian, Ruan CanHua, Shu ZhaoGang, explore desktop virtualization NCRE application [J] Experimental Technology and Management, 2014,03: 118-121 + 142

[10] Breivold, Hongyu Pei; Crnkovic, Ivica Cloud Computing education strategies,2014 IEEE 27th Conference on Software Engineering Education and Training, CSEE and T 2014 - Proceedings, p 29-38, 2014 